\title{
Inauguration des installations d'essais à trois millions de volts du Laboratoire Ampère
}

Le 21 juin, en présence d'une nombreuse assemblée de savants et de chefs d'industrie, ont été inaugurécs les nouvelles installations du laboratoire Ampère. Ce laboratoire, construit par la Compagnie Générale d'Electro-Céramique, était, lors de sa première inauguration en 1923, le premier en Europe où la tension de un million de volts entre un pôle et la terre, ait été réalisée. Depuis lors il a rendu des services considérables aux constructeurs de nos lignes de transport d'énergie à haute tension. Les artères principales du réseau qui connecte les centres de production d'énergie électrique et les réunit aux centres principaux de consommation, ont été au cours des dix dernières années, réalisées avec des tensions de fonctionnement de 150.000 ou 220.000 volts : l'étude et la mise au point des isolateurs capables d'assurer l'isolement à la terre de tensions aussi élevées, n'ont été possible que grâce aux recherches effectuées dans les laboraloires du genre du laboratoire Ampère.

Celui-ci a été, dès sa construction, un instrument de recherches incomparables et a puissammant contribué au développement de la technique de I'isolement électrique : nous rappellerons plus loin de quels équipements d'essais électriques, mécaniques el thermiques il a été doté à l'origine.

Mais les problèmes de 1933 sont plus complexes que ceux de 1923 ; on n'envisage certes pas la réalisation de lignes à plus de 220.000 volts : la tension d'essais de un million de volts est largement suffisante pour l'étude des isolements actuels. Mais il est d'autres phénomènes qui provoquaient déjà sur les rẹseaux des incidents très gênants et dont la nature était à peu près inconnue; je veux dire les phénomènes dus aux orages et à la foudre. Le perfectionnement ou la création de nombreux appareils comme l'oscillographe à rayons cathodiques de Dufour, les recherches systématiques faites à grands frais sur des réseaux américains et suisses, ont permis d'analyser les manifestations de la foudre et de déterminer la nature et l'importance des troubles qu'elle produit dans les installations; la foudre tombant sur une ligne y détermine un accroissement de la tension, dite surtension, qui peut dépasser deux millions de volts; la raison pour laquelle il n'était pas possible de la mesurer, est qu'elle est transitoire; il arrive qu'elle survienne en moins d'un millionième de seconde, et qu'elle disparaisse après dix millionièmes de seconde. Ces surtensions si courtes ont une grosse importance, parce qu'elles peuvent déterminer sur les lignes des courts circuits qui les mettent hors service et faire subir aux isolateurs des contraintes anormales.

Les isolateurs modernes convenablement protégés supportent ces contrainles habituellement sans fatigue mais il est devenu indispensable de connaître leur action el de l'étudier avec soin.

Ce préambule a pour but d'expliquer pourquoi la Compagnie Générale d'Electro-Céramique a été amenée à développer les installations du laboratoire Ampère ; aux installations existantes qui restent fondamentales elle a ajouté une installation permetlant de réaliser dans une enceinte fermée et obscurc, de vérilables éclairs de foudre éclatant à des tensions pouvant atteindre 3 millions de volts: les surtensions sont produites à des vitesses réglables à volonté; loute surtension observée dans la réalité peut être reproduite identique en laboratoire et une fois repro- duite, enregistrée grâce à ce merveilleux appareil l'oscillographe à rayons cathodiques de l'inventeur français Dufour.

La Compagnie Générale d'Electro-Céramique a profitè des modifications qu'elle a dû faire subir aux dispositions intérieures du laboratoire Ampère pour perfectionner les installations anciennes suivant les enseignements de son expérience décennale. Ce laboratoire dont nous avons visité les principales installations est à l'heure actuelle le mieux équipé d'Europe pour les essais à haute tension.

C'est à Ivry que ce laboratoire est édifié. Le bâtiment est une énorme cage métallique de 36 mètres de long, 20 de large, 18 de haut, au milieu de laquelle on a posé, sur des socles en béton, trois énormes transformateurs ainsi que le générateur de foudre.

Il n'y a pas de fenêtre, l'obscurité étant nécessaire pour l'observation des ares et des moindres aigrettes lumineuses qui sont émis par les corps sous tension.

Une vaste baie de 12 mètres de côté, garnie d'un rideau de fer, y est ménagée pour le passage des lignes électriques, lorsque l'on désire faire des expériences à l'extérieur.

C'est là que les invités à l'inauguration ont été reçus par le. Président du Conseil d'administration de la C. G. E. C., M. le Professeur d'Arsonval, dont le jubilé a été récemment célébré en Sorbonne, en présence du Président de la République.

Avec son affabilité coutumière, le Professeur d'Arsonval a présenté à ses hôtes les installations anciennes et nouvelles du láboratoire.

D'abord l'installation de 1923 , avec ses trois transformateurs permettant d'obtenir la tension de un million de volts en courant alternatif normal, ses sphères de un mètre de diamètre pour la mesure de la tension, sa cuve à huile de 30 mètres cubes de capacité, pour les essais de perforation, ses machines d'essais mécaniques sous tension électrique.

Ces appareils n'ont pas été changés depuis 1923, mais sculement perfectionnés sur des points de détail.

Le clou de l'inauguration était l'installation de foudre artificielle : la nature produit la foudre en chargeant électriquement des nuages, l'éclair jaillit lorsque la différcnce de potentiel entre des nuages ou entre un nuage et le sol, a atteint une valeur suffisante.

Au laboratoire Ampère ce sont des condensateurs empilés qui jouent le rôle de nuages. On les charge électriquement au moyen d'un générateur de courant continu à haute tension; ce temps de charge dure plusieurs secondes; brusquement un arc jaillit, assourdissant comme un coup de canon ; c'est un tronçon d'éclair, il en a les mêmes caractéristiques et les mêmes effets. Pour mesurer le potentiel de ces arcs on se sert de sphères de deux mètres de diamètre, entre lesquelles on fait jaillir l'étincelle à mesurer et on enregistre l'oscillation produite au moyen d'un oscillographe à rayons cathodiques.

Devant les invités, diverses expériences ont été faites : chute de la foudre sur un tronc d'arbre qu'elle a déchiqueté, passage 
de l'éclair dans un récipient d'eau, passage de l'éclair le long d'une chaîne d'isolateurs, éclairs entre pointes écartées de plus de 4 mètres;

Les arcs réalisés représentaient la décharge en quelques millionièmes de seconde, d'une puissance de l'ordre de 10 millions de chevaux, soit plus de deux fois la puissance totale des usines génératrices françaises.

Les invités ont ensuite visité en détail toutes les installations annexes du laboratoire, la station d'essais de durée où des isola- teurs sont installés depuis plusicurs ammées, dans des conditions de fatigue identiques à celles qu'ils trouvent en service, les machines d'essais mécaniques à la traction, à la flexion, les cuves d'essais de température, enfin la salle d'exposition où sont rassemblés tous les produits les plus récenls de la Compagnie Générale d'Electro-Céramique, depuis les petites poulies en porcelaine de quelques millimètres de diamètre, jusqu'aux pièces de traversées de deux mòtres de haut.

P. P.

\title{
Congrès International d'Electricité - Paris 1932
}

\begin{abstract}
Il a semblé, à la Houille Blanche, utile pour ses lecteurs de leur donner ici les analyses de quelques rapports particultèrement intéressants, prèsentés à l'occasion du Congrès International d'Electricité de
\end{abstract} 1932.

On sait l'importance de cette manifestation, liée au Cinquantenaire du premier Congrès International d'Electricité de 1881, à Paris. Parallèlement à des cuvres de progression méthodique (comme celle de la Conférence Internationale des Grands Réseaux, qui se tient à Paris tous les deux ans, pour ne citer qu'elle), le Congrès International de 1932 s'était donné pour.mission, dans ses treize sections, de produire des mises au point, et mème des exposés complets de l'évolution, durant ces cinquante ans, de certaines des questions électrotechniques qui jouissent d'assez de recul pour motiver un tel retour en arrière. Pour d'autres plus jeunes, comme celle de la Physique Nucléaire, aux progrès si étonnamment rapides et aussi à la marche parfois si déconcertante, d'intéressants rapports constituent des monuments définitifs, autant qu'ils peuvent l'être en de si fluctuantes matières. Dans la première série de ces analyses, sont examinées, parmi les plus importantes, quelques-unes des communications du Congrès relatives à la traction électrique.

\section{Evolution et développement de l'électrification à courant continu dans les chemins de fer européens}

par M. Cardon.

$$
5^{\text {e SECTION. - RAPPORT N }} 3 \text { (FRANCE) }
$$

Les installations envisagées dans ce rapport sont surtout les électrifications à 1500 et $3000 \mathrm{~V}$. employées en Europe depuis 1918, en France (Compagnies du Midi, du P. O. du P.-L.-M.), au Maroc, en Espagne, en Algérie et en Tchéco-Slovaquie.

L'auteur examine d'abord la partie mécanique, et ensuite l'équipement électrique.

Ces installations ont donné toute satisfaction, grâce surtout à leur entretien très réduit, en dépit de la dureté du service.

PREMIERE PARTIE

\section{DISPOSITIONS GÉNÉRALES DES LOCOMOTIVES}

L'auteur a classé les locomotives en trois catégories : locomotives à grande vitesse, mixtes à voyageurs et marchandises et de manouvre.

1. Locomotives à grande vitesse. - Ce sont celles dont la vitesse normale dépasse 80 à $90 \mathrm{~km} / \mathrm{h}$. La diversité des types de machines peut s'expliquer par le manque d'expérience qu'éprcuvaient les constructeurs jusqu'ici dans ce genre de locomotives.

2. Disposition des caisses et des trains de roues. - Toutes ces machines présentent d'abord un caractère commun : la symétrie. D'habitude, on a deux cabines : une à chaque extrémilé. On connaît des locomotives à une ou à deux caisses. Avec une scule caisse, on a deux solutions de la suspension : disposition à châssis rigide, ce qui limite, à cause des courbes, à quatre le nombre d'essieux, donc le poids adhérenl, mais offre par conlre une irréprochable lenue en ligne aux grandes vitesses dépassant 130 à $140 \mathrm{~km} . / \mathrm{h}$, et disposition de deux trucks attelés cnsemble, qui a permis de porter à six le nombre d'essieux, augmenter ainsi le poids adhérent, l'effort de traction, donc la puissance. Mais, la tenue en voie est moins bonne; à partir de $110 \mathrm{~km} . / \mathrm{h}$., on observe des mouvements de lacets. Les locomotives à deux caisses sont à rapprocher à ce dernier type et suscitent les mêmes remarques. On peut en conclure que les dispositions rigides à caisse unique conviendront particulièrement bien aux très grandes vitesses.

3. Suspension des moteurs. - Sauf une exception, les locomotives Gearless, dans lesquelles les induits sont calés directement sur les essieux, n'ont pas assuré encore un parcours assez important, pour qu'on puisse juger leurs qualités. Toutes les locomotives à grandes vitesses ont leurs moteurs entièrcment suspendus.

4. Liaison déformable entre moteurs suspendus et essieux. La nécessité d'une telle liaison avec les moteurs suspendus est évidente. Elle se fait par bielles (système Kando, par exemple) ou par engrenages avec liaisons déformables.

Les roues d'engrenages peuvent être à l'intérieur ou à l'extérieur des roues motrices. On a un ou deux trains d'engrenages. La transmission du mouvement de l'engrenage à la roue se fait de différentes façons : à ressort, par bielleltes articulées, par biellettes et secteurs dentés (système Büchli de la B. B. C.), par transmissions élastiques (Oerlikon). Les axes des moteurs sont le plus souvent horizontaux; on les voit dans quelques cas exceptionnels verticaux, ce qui permet d'élever le centre de gravité, done d'améliorer la stabilité, mais complique la construction et ne permet pas une grande puissance par essieu. La transmission par engrenages est beaucoup plus employée que les bielles, grâce à leur facilité d'entretien. L'avanlage des bielles est surtout une adhérence parfaite, mais ce qui leur fait préférer les engrenages, c'ost leur entretien délicat et leur montage plus difficile. 